\title{
On the trail of the hippocampal engram
}

\author{
JOHN O'KEEFE and DULCIE H. CONWAY \\ Cerebral Functions Group, Anatomy Department and Centre for Neuroscience \\ University College London, London WCIE 6BT, England
}

\begin{abstract}
Recent ideas about hippocampal function in animals suggest that it may be involved in memory. A new test for rat memory is described (the despatch task). It has several properties which make it well suited for the study of one-trial, long-term memory. The information about the location of the goal is given to the rat when it is in the startbox. The memory formed is long lasting $(>30 \mathrm{~min})$, and the animals are remembering the location of the goal and not the response to be made to reach that goal. The spatial relations of the cues within the environment can be manipulated to bias the animals towards selection of a place hypothesis or a guidance hypothesis. The latter does not seem to support long-term memory formation in the way that the former does. Fornix lesions selectively disrupt performance of the place-biased task, a finding that is predicted by the cognitive map theory but not by other ideas about hippocampal involvement in memory.
\end{abstract}

Since 1957, when Scoville and Milner (1957) reported that bilateral removal of the mesial temporal lobes in man resulted in a profound global amnesia, it has generally been accepted that the hippocampus plays an important role in human memory (but see Horel, 1978, for an important paper questioning this dogma). It is tempting therefore to search for an explanation of the changes in animals' behavior following hippocampal damage in terms of a memory deficit. Three recent ideas about the function of the hippocampus in animals have suggested just such a memory function (Gaffan, 1972, 1974; Olton, Becker, \& Handleman, 1979, 1980; O'Keefe \& Nadel, 1978). All three have in common the idea that there is more than one kind of memory and that the hippocampus is involved in only one of these. They differ in the types of memories envisioned. Gaffan thinks there are distinct memory systems for recognition and association and that the hippocampus is concerned only with recognition memory. Olton draws a distinction between working memory and reference memory. The former is required in tasks where the response to a particular stimulus varies from trial to trial, while the latter is suitable for tasks where correct performance consists of the same response to a stimulus across trials. Only working memory requires an intact hippocampus.

We have also distinguished between two memory sytems and have assigned different properties to them. The locale system provides the animal with a mapping system which enables it to locate itself in an environment and to generate place hypotheses about that environment. The representation of a place is formed (or changed) in an all-or-none fashion on the basis of a single exposure to a part of an environment, and there

The lesion experiments described in this article were done in collaboration with Francoise Schenk and were supported by the Medical Research Council of Great Britain. is little interference between different place representations. Consequently, the mapping system should be capable of supporting high levels of performance in tests of animal memory, such as the delayed reaction task of Hunter (1913).

In contrast, the other memory systems, the taxon systems, store information in such a manner that it cannot easily be used by an animal in the delayed reaction task. These systems enable an animal to solve a problem by approaching or avoiding specific cues (guidance hypotheses) or by performing specific responses at choice points (orientation hypotheses). Information is stored in these systems in an incremental fashion, and there is considerable interference among similar representations. Thus, one would not expect high performance from an animal using one of these taxon hypotheses in a delayed reaction task. We will return to a discussion of these points toward the end of this paper.

In order to study the role of the hippocampus in different types of learning and memory, we have devised a behavioral task in which we control the extramaze cues that the rat uses to find its way to the goal. By manipulating the spatial relations among these cues and the goal, we have tried to bias the animal toward adopting either a place hypothesis or a guidance hypothesis. In one condition, the cues are distributed around the environment and should be most easily used to locate the position of the animal and the goal in the environment (distributed-cue condition); in the second condition, the cues are all concentrated behind the goal and should be most easily incorporated into a guidance hypothesis where the animal directly approaches one or more cues (clustered-cue condition).

In the first part of this paper, we will discuss evidence from experiments using the distributed-cue task which indicates that normal rats have a good long-term memory for places. Some of the properties of this memory will 
be discussed. In the second part of the paper, we will describe the results of an experiment in which normal and fornix lesioned rats were tested on place and guidance tasks. Several conclusions can be drawn from these results. Most important of these are, first, that there appears to be a close connection between place learning and long-term memory and, second, that lesions of the fornix have a greater effect on place learning than on guidance learning.

\section{The Characteristics of an Ideal Memory Task}

Following William James (1890), we will define memory as "the knowledge of an event, or fact, of which meantime we have not been thinking, with the additional consciousness that we have thought or experienced it before" (Vol. 1, p. 648, emphasis in the original). ${ }^{1}$ Thus the acquisition of a habit or a conditioned response does not automatically qualify as a memory unless it can be shown that the animal also has knowledge of the event. On this definition it is possible to have learning without memory. It is also possible that animals do not have memory at all.

In order to test memory, we need a task that has three phases: a registration phase, during which the event is perceived or the information acquired by the animal; a retention phase, or interval following the event during which the information cannot be acquired but must be stored by the organism; and a retrieval phase, during which the animal must use the information for some purpose or other. The task should be repeatable so that the memory capacity of the animal can be assessed at different retention intervals and the properties of the trace assessed through the use of different probe tests. Although this can be attempted using different groups, it is probably unwise, since different animals may store the same event in different ways.

If the task is to be used for the study of the neural basis of memory, there are several additional criteria which should be satisfied. First, there should be a perceptual counterpart to the memory task in which the registration and retrieval phases are collapsed so that the effects of any brain or behavioral manipulation on the animal's ability to remember the event can be separated from its ability to perceive that event. Second, the amount of time given to the different phases should be under the control of the experimenter and not the animal. It is known, for example, that increasing the length of registration time has an effect on the delay period over which the information can be retained in some memory tasks (Alexinsky \& Chapouthier, 1978). Third, the registration of the information should depend as little as possible on the animal's behavior and should occur in a different place and under different circumstances from those of the information retrieval phase. This is to preclude simple conditioning effects of the brain or behavioral manipulation during the retention phase on the animal's behavior during the retrieval phase. Thus, if an animal is fed in a place during the registration phase and required to return to that place after a delay during the retrieval phase, brain manipulation during the interval may change the animal's desire to return to the place depending upon whether that manipulation is painful or pleasureful. The usual attempt to obviate this difficulty is through the use of avoidance tasks in which the brain intervention is thought to be aversive (Coons \& Miller, 1960). The best example of this is the use of the one-trial step-down avoidance experiment and ECS (McGaugh \& Herz, 1972). But it is dangerous to assume that any treatment is aversive or pleasant or that the same treatment might not be noxious for some animals and pleasant for others or have different affective properties for the same animal at different times. Better to reduce the problem to a minimum or avoid it altogether if possible. For these and other reasons, the indirect delayed reaction task (DRT) is preferable to the direct delayed reaction task. In the indirect DRT, the animal is given the information during the registration period while it is in the startbox and without its having to make any response other than an observing one. In the direct DRT, the animal must make a response to the correct cue or else is placed in the goalbox, and any subsequent manipulation may influence its attitude toward that cue or place. The task described in this paper is an indirect delayed reaction task and, as such, seems well suited to the study of the neural basis of memory in the rat.

\section{The Rat's Memory for Places}

In many memory tasks designed for the rat, places are available as retrieval cues but are not used as the primary registration cues. For example, in Hunter's original delayed reaction task, a light was used during the registration phase to signal which of three compartments the rat should approach during the retrieval phase (Hunter, 1913). One can think of the light (in a place) as the registration information and the place alone as the retrieval information. We (O'Keefe \& Conway, in preparation) wanted to use a task where the place could be used as the primary registration information. In order to do this, we needed to control the cues which an animal used to locate itself in an environment. The task we ended up with grew out of previous attempts to control the place cues in a study of the hippocampal place cells (O'Keefe \& Black, 1978; O'Keefe \& Conway, 1978).

The layout of the testing situation is shown in Figure 1. An elevated + maze was centered within a set of black curtains measuring $7 \mathrm{ft}$ on each side. Inside this enclosure there were six cues which the rat could use to locate itself and the goal. They were a dim light, a large white card, a fan, a wire cage containing two rats, a black towel, and an aromatic marker pen. The animals were taught a discrimination based on these cues using 


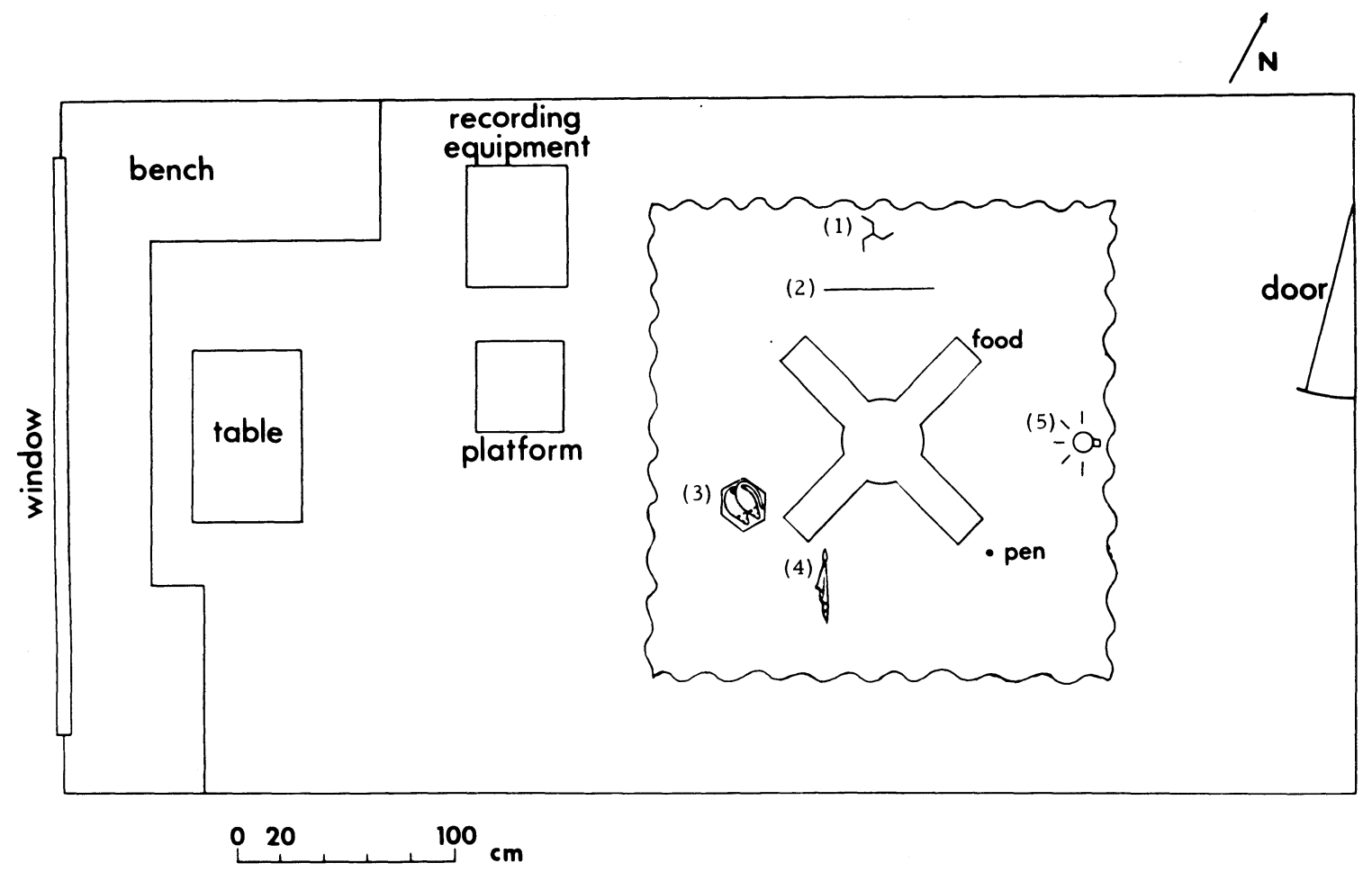

Figure 1. A ground plan of the experimental room showing the cue-controlled environment curtained off from the rest of the room. The symbols inside the room represent the following: at location (1), fan; at (2), large white card; at (3), cage with two rats; at (4), towel; at (5), light. Food marks the goal.

a procedure similar to the one previously described (O'Keefe \& Black, 1978; O'Keefe \& Conway, 1978). Briefly, a constant spatial relationship was maintained among the cues and the goal throughout the experiment, but these were rotated as a constellation by $90 \mathrm{deg}$ or multiples of $90 \mathrm{deg}$ from trial to trial (see Figure 2). Other strategies for solution of the problem were ruled out, since the maze itself was not rotated between trials and different body turns were required to reach the goal on different trials. We call this controlled-cue memory task, the despatch task, as a shorthand for delayed spatial choice task.

Rats learn the perceptual discrimination inside the cue-controlled environment rapidly. In our overall series of 41 normal animals trained under a variety of conditions (with or without correction, different amounts of pretraining), only 5 animals required more than 30 trials (10 trials/day) to reach the criterion of 9 correct in 10 successive trials. This suggests that most rats find this task simple to learn and are presumably fooled into treating it as a place learning task in a stable environment. For the purposes of the first part of this paper, we will concentrate on the behavior of a group of 8 normal rats in which we explored the properties of the memory associated with this learning.

Seven of the eight rats learned the perceptual task in a median number of 7 trials to criterion (range 5-34).
The other rat is the only animal that has thus far failed to learn the place discrimination in a reasonable number of trials, and was dropped from the study after 68 trials.

The seven rats that learned the perceptual task were tested on the memory task. The memory procedure is shown in Figure 3 (bottom, despatch trial). At the beginning of the trial, the rat was confined to the start arm and allowed to register the positions of the controlled cues. Before the animal was allowed to run, the cues were removed (Step 2) and, either immediately or after a short delay (Step 3), the rat was allowed to choose (Step 4). The delay was determined by a titration procedure, starting at the minimal time required to remove the cues and increasing as the animal achieved a predetermined level of performance. Failure at longer delays was followed by a return to a shorter delay. Six of the seven rats that had reached criterion on the perceptual task learned to perform the memory task with a high success rate ( 9 correct in 10 trials irrespective of delay) in a very small number of additional trials (median $=2$; range $0-5$ ). The seventh animal failed to reach criterion on the memory task in 27 trials and was dropped from the study. In general, we find that approximately one in every four animals which learns the perceptual task fails to perform well on the memory task. Possible reasons for this will be disclussed below. 
A

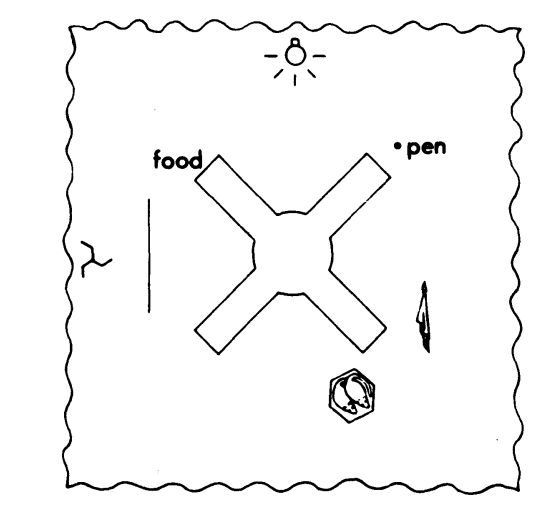

B
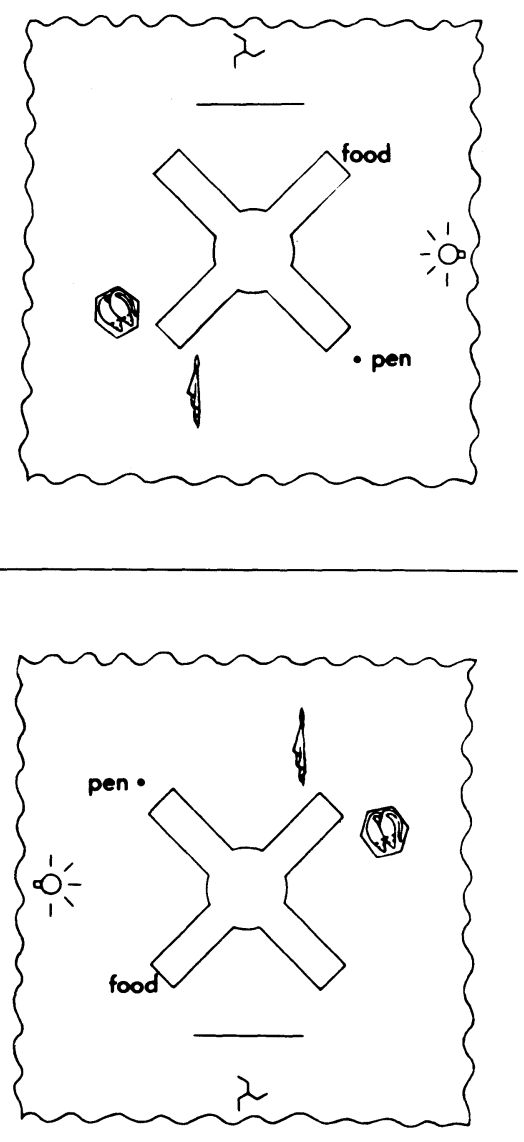

Figure 2. Four orientations of the cues and goal relative to the rest of the experimental room. Each position represents a 90 -deg clockwise rotation relative to the previous position. At each orientation, the rat was started from a different nongoal arm on different trials so that it could not solve the task by consistently making the same body turn at the choice point.

\section{Properties of the Memory Trace}

Concentrating on the six rats that learned the memory task, we asked a series of questions about the properties of the memory. First, we wished to know whether there was any significant decrease in the animal's ability to perform the task as the delay interval between removal of the cues and the animal's choice was increased. In his original delayed reaction experiments, Hunter (1913) found that the rats could perform correctly only with short delays up to $10 \mathrm{sec}$. Subsequent experiments (e.g., MacCorquodale, 1947; McCord, 1939) extended this interval to $4 \mathrm{~min}$, and there were some reports of much larger intervals (e.g., Maier, 1929). More recent experiments have reported above chance performance with delays of $50 \mathrm{~min}$ (Olton, 1972), $135 \mathrm{~min}$ (Sinnamon, Freneire, \& Kootz, 1978), and 24 min (Alexinsky \& Chapouthier, 1978).

Figure 4 shows the results of extending the delay in the present task. Each point represents the mean performances of the six animals on the last block of trials. For most points this represents seven trials, but for a few points only five trials were given altogether. It is clear that, for all animals, performance remains close to perfect, even up to delays of $30 \mathrm{~min}$. As can be seen from the range scores, no animal scored lower than 5 correct in 7 trials, and thus all points for all animals are significantly different from chance at the .05 level or better. It is clear that the trace laid down during the brief exposure to the controlled cues at the beginning of each trial is a powerful, long-lasting one. Unfortunately, the high level of performance does not enable us to rule out the possibility that there is some change in the underlying trace over the intervals tested, nor can we rule out the possibility that the animal's level of performance would begin to fall off if the delay were made long enough.

\section{Control Trials}

Could the animals have been using some cue other than the remembered position of the controlled cues to locate the goal during the retrieval phase? We eliminated the food as a cue by baiting the goal arm only after the animal had made its choice (delay of reward). But we worried that physical traces of the controlled cues 


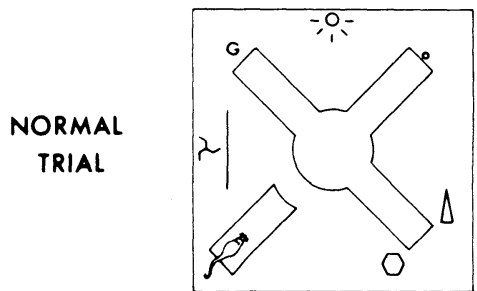

rat in start

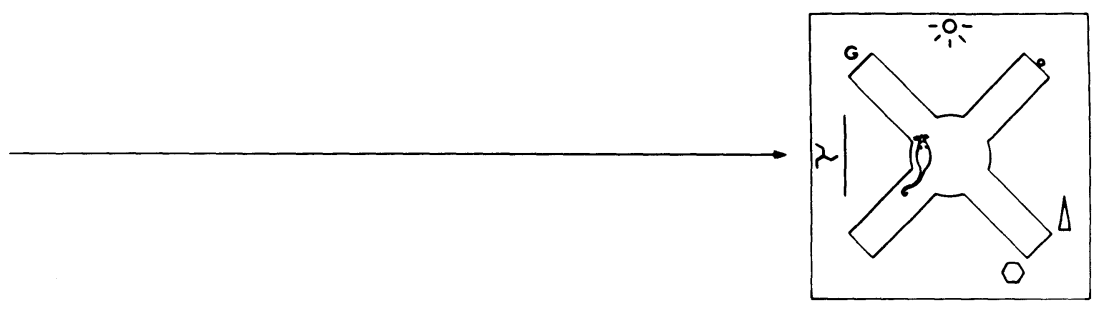

rat chooses

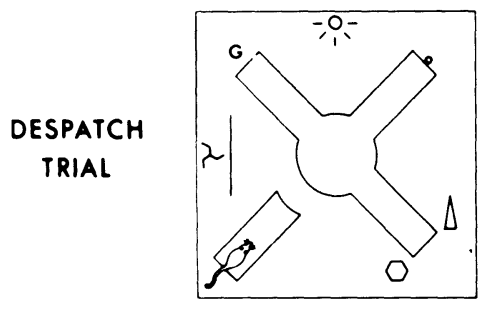

rat in start

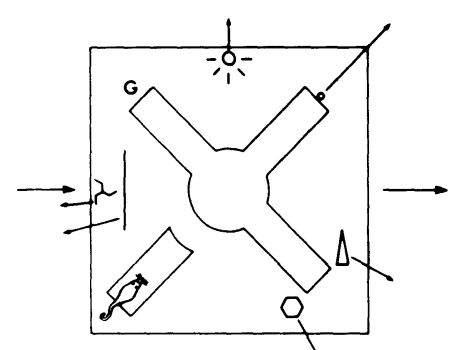

remove cues

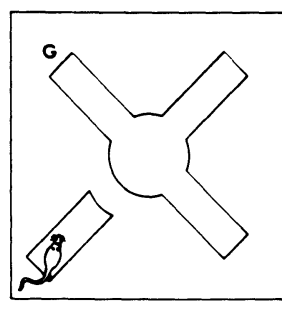

delay

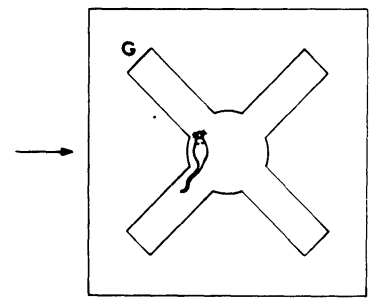

rat chooses

Figure 3. Schematic diagram showing the successive steps in a normal perceptual trial (top) and a despatch memory trial (bottom).

might remain after their removal and that the rat might use these to locate the goal. For example, the most recently illuminated bulb might remain warmer than the others for some time after being extinguished, or subtle air currents might continue to circulate within the enclosure for considerable periods after the fan was switched off. We also wanted to control for experimentergenerated effects. At the extreme, we considered the possibility that the rats were reading our minds during the delay. More plausibly, they might have been cued by the experimenter's behavior during the various points of the procedure at which we interacted with them (in particular, when the arm was being pushed in at the end of the delay period).

The contribution of these sources of artifact was assessed during control trials (Figure 5). These were

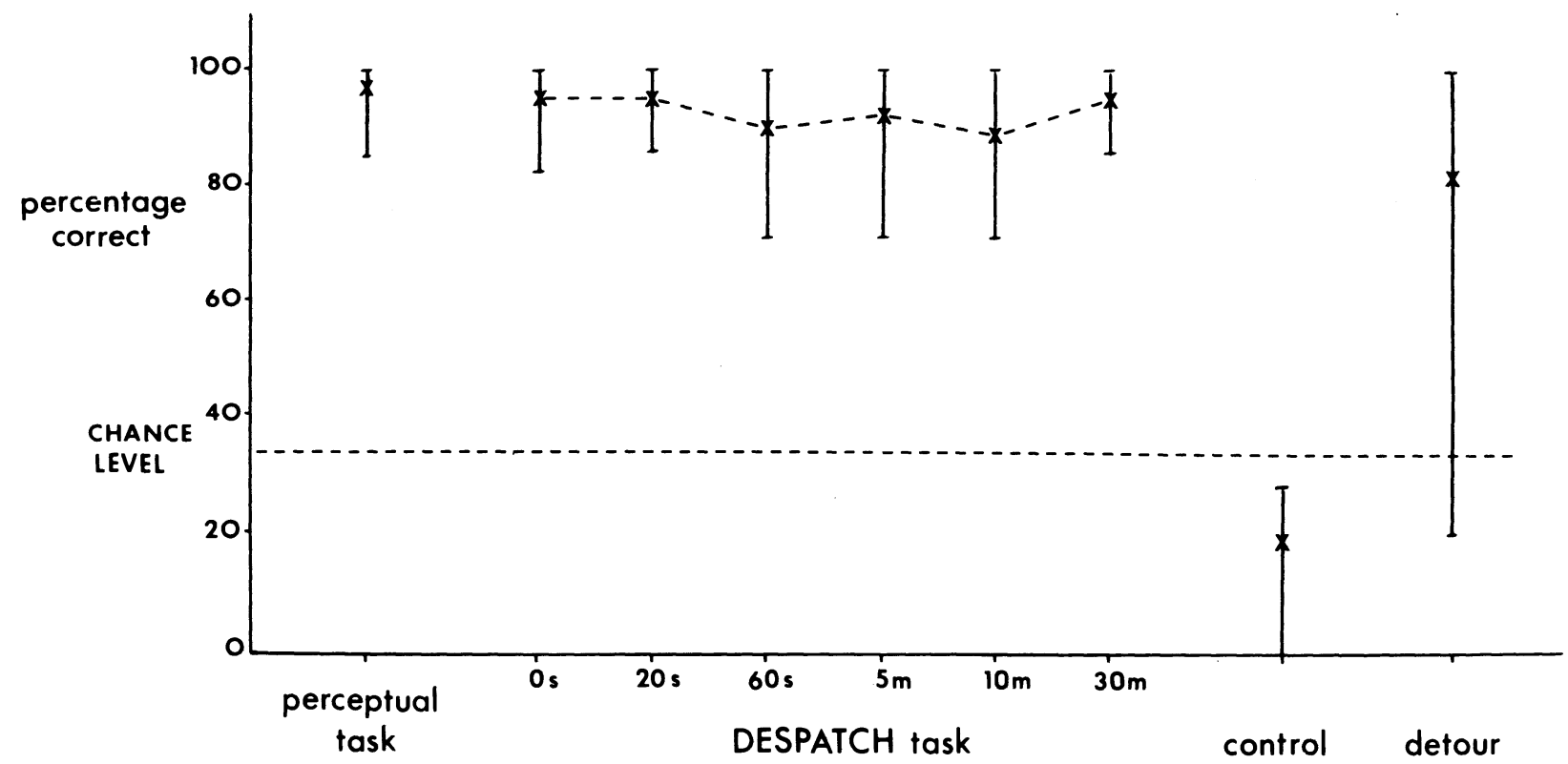

Figure 4. Asymptote performance of six normal rats on the despatch task during delay, control, and detour trials. The scores on the perceptual trials were taken from the second normal perceptual trial on those days when delay trials were given. The bars represent the range of scores of the animals. The delay time was calculated from the point at which the last cue was removed from the environment. The average time to remove the cues was about 15 sec. The large range score during the detour trials is due to one animal, which refused to do the task and scored 1 correct in 5 before giving up. 
DESPATCH TRIAL

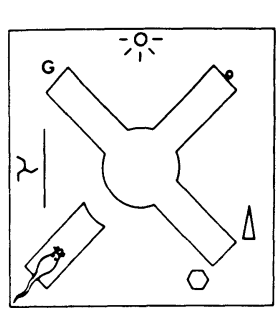

rat in start

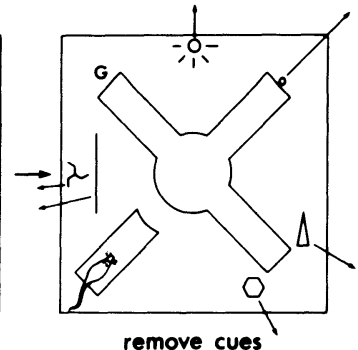

remove cues

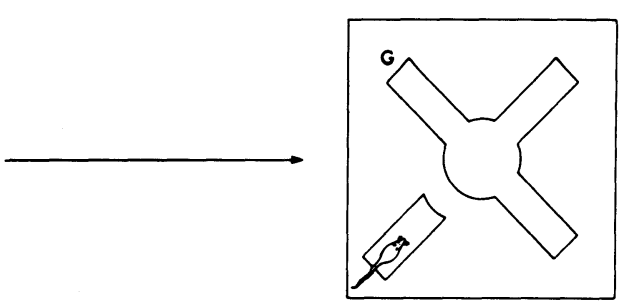

delay

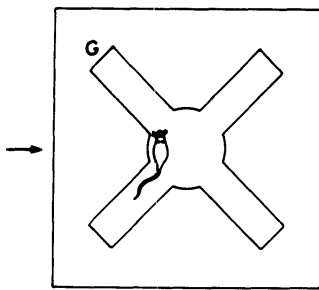

rat chooses

\section{CONTROL TRIAL}

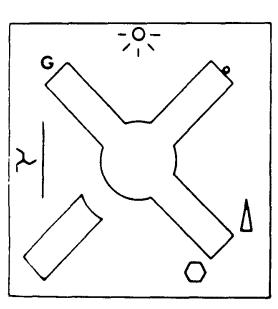

cues in position

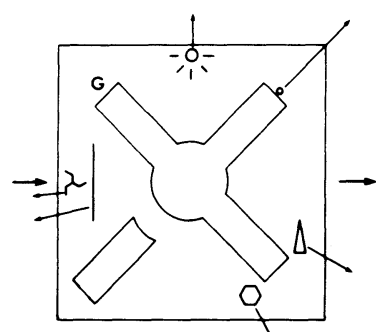

remove cues

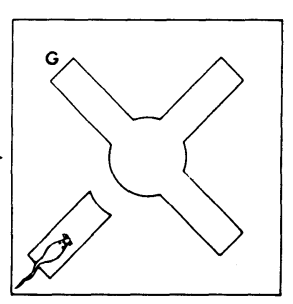

rat in start

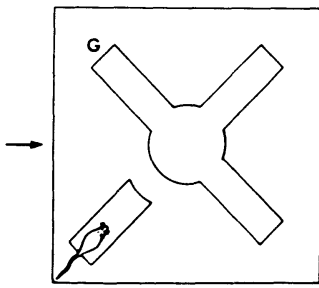

delay

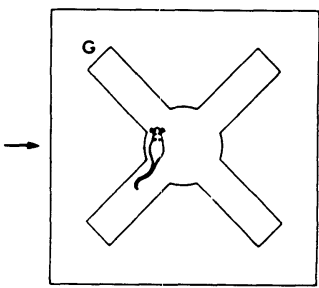

rat chooses

Figure 5. Schematic diagram showing the procedure during a control trial (bottom) as compared with a despatch memory trial (above). The important difference is that the animal is placed in the start arm at the beginning of the despatch trial, but only during the third step of the control trial, after the cues have been removed.

identical to the standard despatch trial except for the point in the procedure when the rat was placed in the start arm. In the standard despatch trial, the rat was there from the start and was thus exposed to both the cues and their aftereffects. In the control trials, the rat was introduced into the environment only after the cues had been removed so that it was exposed only to the aftereffects of the cues and not to the cues themselves (Figure 5, bottom, Step 3). Furthermore, the experimenter's knowledge and behavior were identical during the two trials, only the sequence of events was changed. The performance of all six rats fell to chance level during these control trials (Figure 4, control).

\section{What Does the Rat Remember?}

The testing procedure is flexible enough to enable us to ask what the rat is remembering during the delay. Hunter (1913) noted that his successful rats oriented towards the correct goal box during the registration period and maintained that orientation during the delay. Our rats did not appear to do this but, instead, often ran back and forth in the start arm during the delay. Nevertheless, they might have calculated the body turn required to reach the goal during the registration phase and committed that to memory. The way to test this is to prevent the rat from knowing, during the registration phase, what body turn it will be required to make in order to reach the goal during the retrieval phase. The procedure is shown in Figure 6. The first three steps of the detour trial are identical to a despatch trial in that the animal is confined to the start arm for a period of time and allowed to perceive the controlled spatial cues which are then removed. After the 1-min delay, however, the rat is not allowed to choose the goal arm. Instead, he is forced to enter one of the two nongoal arms. When he has done so, the two remaining arms are pushed to the center so that he is faced with a choice of three arms. Thus, on any given trial, the rat might be required to make any one of three body-turn combinations in order to reach the goal.

For example, in the spatial configuration shown in Figure 6, he might be required to make a free-choice left (top), or a forced right, followed by a free-choice straight (bottom), or a forced straight followed by a free-choice right. Five of the six rats performed well on this task, scoring $90 \%$ correct at asymptote performance. The sixth animal was upset by the procedure and returned to the start arm on most of the detour trials. He stopped running after five detour trials, at which point his score was 1 correct in 5 trials and testing was discontinued. Figure 4 (detour) shows the performance of the six animals. From these data, it seems clear that most of the rats were not remembering the body turn during the delay. This strategy cannot be ruled out for the sixth animal, however.

These detour trials suggest that most of the animals were remembering something about the location of the goal on each trial. We have done some preliminary experiments on two of the rats in an attempt to see if this information related to features of the environment which remained after the controlled cues had been 


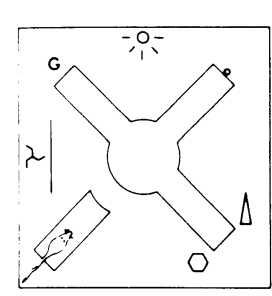

rat in start

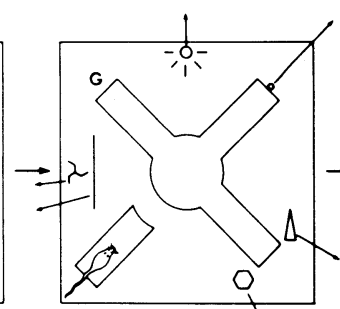

remove cues

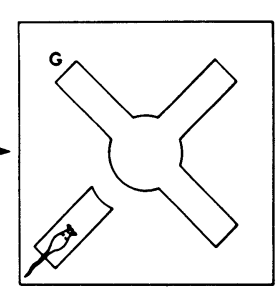

delay

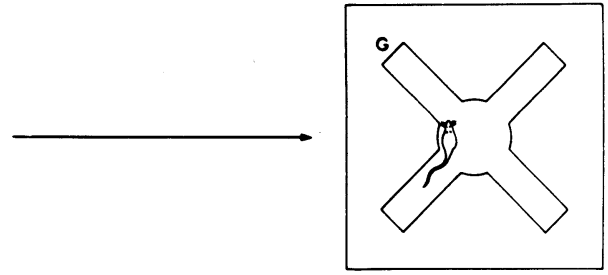

rat chooses

DESPATCH TRIAL WITH DETOUR

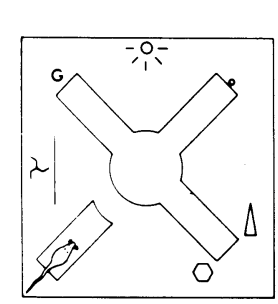

rat in start

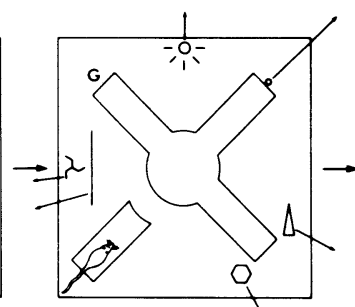

remove cues

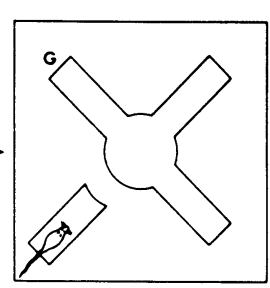

delay

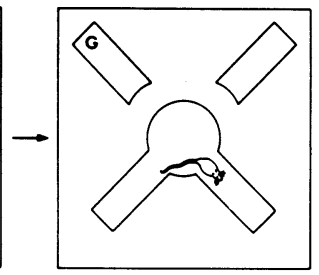

rat forced to run to non-goal arm

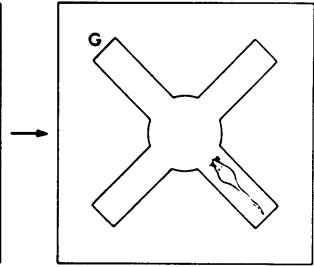

rat chooses

Figure 6. Schematic diagram showing the successive stages in a despatch memory trial with detour (bottom) as compared with a despatch memory trial (top). The important difference is that during a detour trial the animal is not allowed to directly choose the goal arm during the retrieval phase but is forced to enter a nongoal arm (Step 4) before being allowed a free choice (Step 5).

removed ("the goal is towards the sound outside the curtain") or if the memory related the goal arm to the animal's position in the start arm in some way ("It's over there to my left as I face the center but would be straight ahead if I were in that arm over there"). To test this, on some trials the houselights were turned off and the maze was slowly rotated during the delay. We had previously established that turning the houselights off and on during the delay had no effect on performance during the standard despatch task. The maze was rotated at a speed of $1 \mathrm{rpm}$ through 90,180 , or $270 \mathrm{deg}$ in a clockwise or anticlockwise direction. The rats could then choose either the "internal" goal which had rotated with the maze or the original "external" goal as defined by the external surrounds. The results were clear: both rats consistently chose the external goal. This must mean that these rats were establishing a connection between the controlled cues and some cues in the noncontrolled environment inside or outside the curtains during the registration phase and were using the noncontrolled cues as retrieval cues.

\section{The Role of the Hippocampus in Place Memory}

Our group has suggested that the hippocampus is the neural substrate for a cognitive map, that the map generates the information on which place learning is based, and that information can be stored in the mapping system on the basis of a single experience (O'Keefe \& Nadel, 1978). The theory must clearly predict a deficit in the present task following damage to the hippocampal system. Furthermore, it would suggest that a comparable guidance task would not be affected by the same lesion (cf. O'Keefe, Nadel, Keightley, \& Kill, 1975). Recent papers by Olton et al. (1978) and Sinnamon et al. (1978) have shown that lesions of the hippocampus or fornix have a devastating effect on place memory tasks.

Together with F. Schenk, we have done the following experiment to test this prediction. We trained rats on two versions of the cue-controlled task. In the first version, which we call the distributed-cue version, the controlled cues were arranged around the enclosure in the same way as during the previous experiment (see Figure 7A). No cues were directly behind the goal arm. In the clustered-cue version (Figure 7B), the same cues were all placed directly behind the goal arm. Because some of the cues were physically attached to poles set in the middle of each curtain, the maze was rotated 45 deg so that it sat square within the enclosure for this condition. We hoped that the two conditions would be learned through the use of two different hypotheses. As previously suggested, the cues in the distributed-cue condition would be most easily used to locate the animal and the goal in the environment and would form the basis for place hypotheses. The cues in the clustered cue would be less useful as cues for spatial location but could provide the information for guidance hypotheses such as "approach the light (or other cue)." The results were clear. Rats in the clustered-cue condition took significantly more trials (median $=29$ ) to learn the perceptual task than those in the distributedcue condition (median $=13$ ). Furthermore, while 10 of 13 rats in the distributed-cue condition learned the memory task within the limit of 25 trials, none of the 12 
A

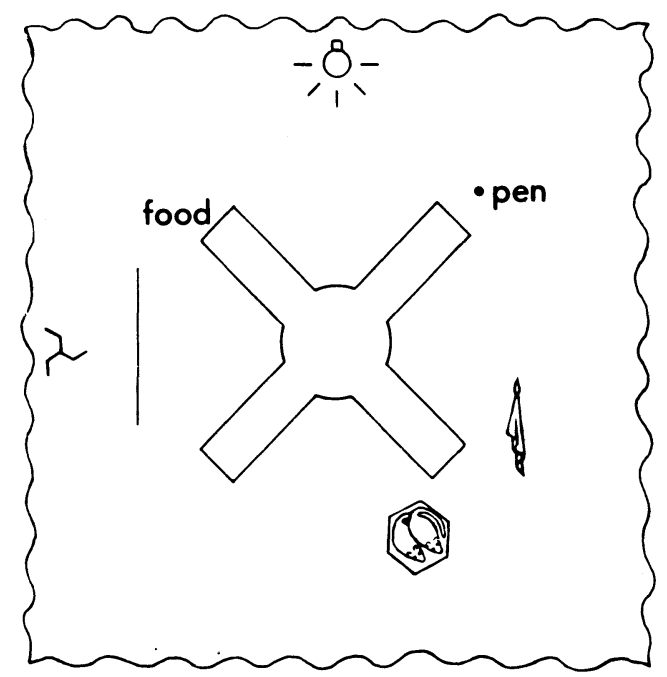

B

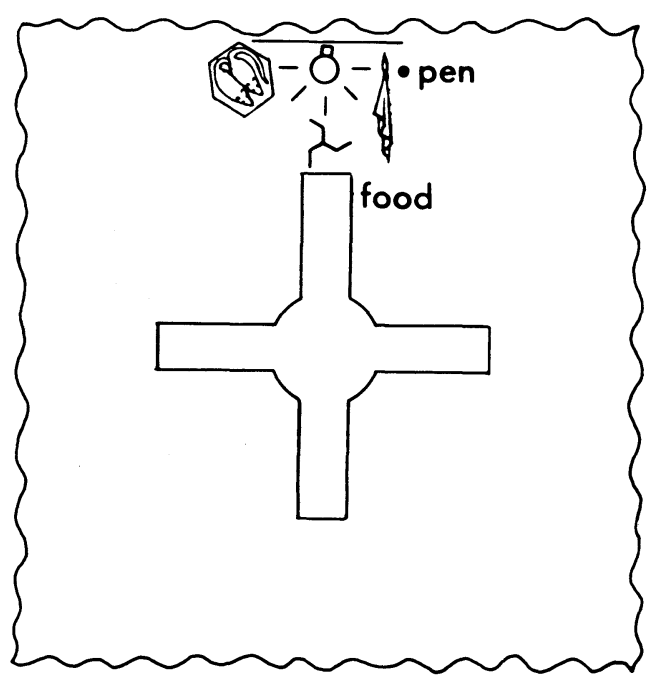

Figure 7. The difference between the spatial layout of the cues on a distributed-cue trial (A) and a clustered-cue trial (B).

rats in the clustered-cue condition learned within this limit. The median number of trials to criterion in the distributed-memory task was 5 .

It would appear, then, that, with this collection of cues, the clustered-cue condition is more difficult than the distributed-cue condition. We do not yet know why this should be so. Furthermore, the clustered-cue condition does not seem capable of supporting memory in the delayed reaction task, at least within the limited training period given in the present experiment. Only 2 of the 12 rats showed above chance performance after 25 trials.

Fornix lesions were made in six rats in each group. The fornix was crushed by watchmaker's forceps inserted into the brain via the lateral approach (see O'Keefe et al., 1975). Three other rats in each group received control lesions. The results of the lesion on the relearning of the perceptual task is shown in Figure 8. While there was no effect on the rats in the clustered-cue condition, there was a devastating effect on the performance of the rats in the distributed-cue condition. Two of the rats failed to relearn in 110 trials and three of the other four showed no savings, taking more trials to relearn than they had required to originally learn the task. Only one of the six animals showed savings, and he was the sole animal in this group that could not originally perform the memory task.

We tested the three rats which relearned the distributed-cue perceptual task in the distributed-cue memory task. None of them relearned the memory version of the task within 70 trials, at which point testing was discontinued (see Figure 9).

It should be noted here that we have repeated the fornix-lesion experiment on animals that had been trained originally on the distributed-cue despatch task with noncorrection training (as above) but were retrained after the lesion using a correction technique in order to reduce the number of trials required to relearn.
These animals did relearn in fewer trials, but still made a large number of errors. In this experiment, two of the five lesioned rats did relearn the memory task. One of

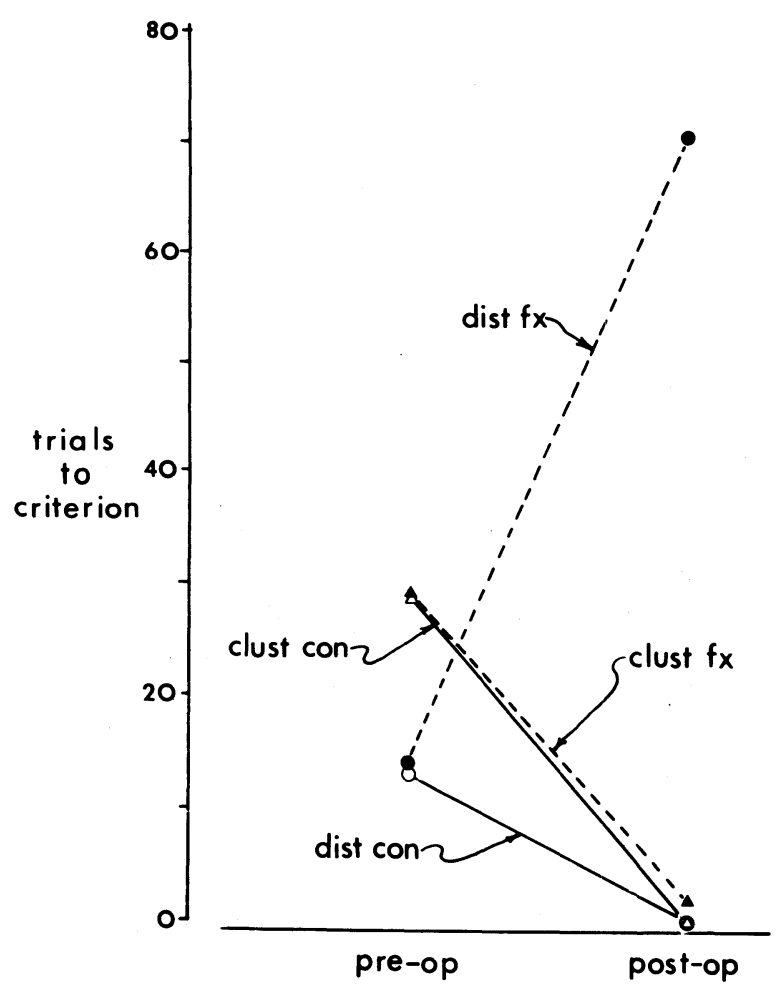

Figure 8. Effects of a fornix lesion on performance of the perceptual phase of the clustered-cue task and the distributed-cue task. The four groups are clustered-cue controls (clust. con.); clustered-cue fornix lesioned (clust. fx.); distributed-cue controls (dist. con.); and distributed-cue fornix lesioned (dist. fx.). Two conclusions are apparent from the graph. First, it takes the clustered-cue group more trials to learn the task originally than it does the distributed-cue group. Second, the fornix lesion has a selective effect on the distributed-cue group. 


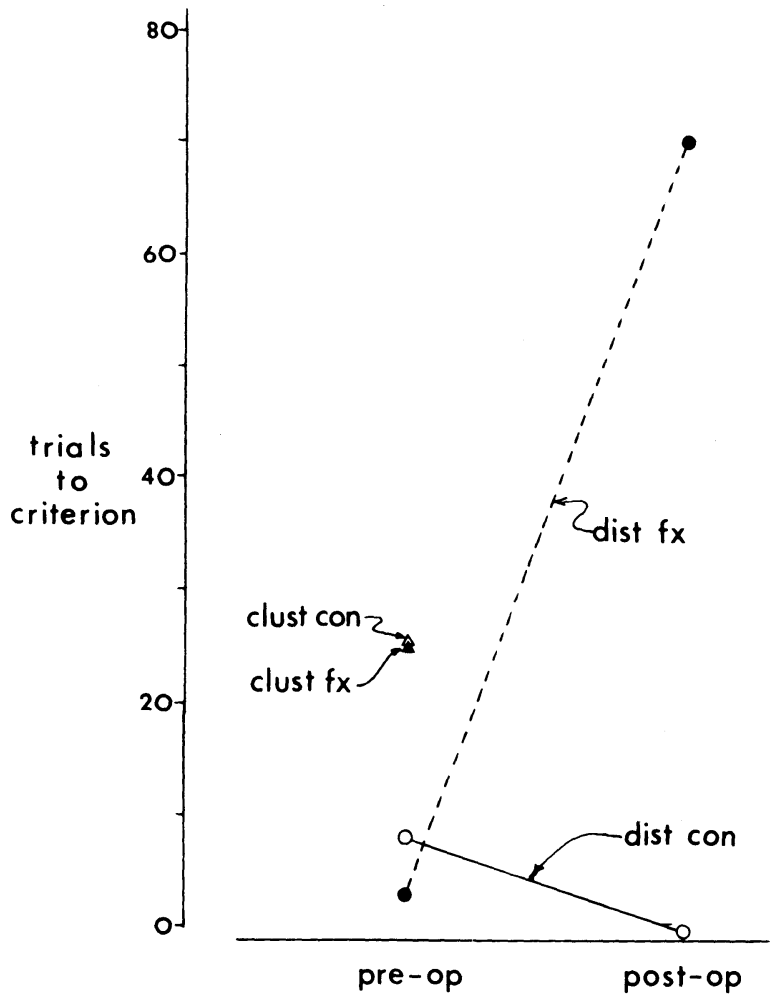

Figure 9. Effects of a fornix lesion on performance of the memory phase for the distributed-cue task group. The groups are the same as in Figure 8. The clustered-cue group failed to learn the memory task preoperatively and were assigned scores of 25 . The postoperative score for the distributed-cue fornix-lesion group represents the three rats that relearned the perceptual task. None of them relearned the memory task within 70 trials, and they were assigned that score.

them took 15 times as many trials to relearn, but the other relearned in one trial! We have no explanation for the behavior of this mischievous fellow. ${ }^{2}$

The pattern of results would seem to admit of the following interpretation. In the clustered-cue condition, the rats are learning to approach one or more of the cues and are using a guidance hypothesis (see O'Keefe $\&$ Nadel, 1978). We have suggested that the learning changes in guidance systems are incremental and subject to interference. Changes strengthen the approach to the cue but would not support behavior in the absence of the cue. Animals using this strategy should not be able to remember the position of the cue longer than a few seconds. In contrast, the distributed-cue condition could be learned using either a guidance hypothesis, "approach one of the cues closest to the goal," or the place hypothesis, "go to that part of the environment as defined by the controlled cues." The place system supports incorporation of new information on the basis of single exposures and would be expected to support memory for the goal position after the removal of the controlled cues. Animals which chose to learn the task using a place hypothesis should quickly learn the memory component. On the other hand, those animals which chose to learn using a guidance hypothesis would not be able to learn the delayed reaction memory task. The differential effects of the fornix lesion on the two perceptual tasks supports this analysis, as does the finding that the perceptual performance of the rat which failed to learn the distributed-cue memory task was only minimally af fected by the fornix lesion. We think that the cuecontrolled environment opens up a set of interesting possibilities. The ability to bias the animal toward one hypothesis or another on the basis of the spatial distribution of the same controlled cues provides a situation for the study of the properties of the different hypotheses. In other tasks, there is always the possibility that the differences between the tasks are due to the selection by the animal of different cues (see Restle, 1957) in the two tasks. In the controlled-cue environment, the set of possible cues is known and postexperiment probes can be used to determine which subset of these cues is actually being used.

The cue-controlled environment enables us to study memory under circumstances in which we have control over the cues during the registration phase. We have not yet identified (or controlled) the cues that the animal uses for retrieval of the information. Experiments in which this is done are under way.

Several lines of experimentation suggest that the hippocampal system is involved in memory for places. First, in this experiment and in previous experiments (e.g., Olton, 1972; Olton et al., 1978; Sinnamon et al., 1978), lesions of the hippocampus or fornix have a marked effect on the animal's ability to perform tasks that involve place memory. The advantage of the present paradigm is that, since it is an indirect delayed reaction task, it incorporates a perceptual component so that it is possible to distinguish between a perceptual and a memory deficit. As we have seen, the deficit following the lesion occurs in both perceptual and memory phases of the experiment, but some animals can relearn the perceptual task. It is not unreasonable to suppose that this relearning was based on a guidance hypothesis which would not support performance in the memory task.

How do the result of these experiments fit with other theories of hippocampal function, in particular the memory theories of Olton and Gaffan? Both the distributed-cue and the clustered-cue perceptual tasks would involve what Olton calls reference memory. In both, the animal is expected to make the same response to the cues on every trial of the test. Thus, the workingmemory/reference-memory distinction would not predict a difference in the effect of a fornix lesion on the distributed- and clustered-cue conditions. On the other hand, the working-memory notion would predict the deficit which was found in the distributed-cue memory task, since this would be classified as a working memory task. Unfortunately, the inability of the rats to learn the clustered-cue memory task within the allotted number of trials prevented us from testing the prediction that there would be a deficit here as well. To do that, 
we will need to find an unambiguous guidance task which supports long-term memory, if such a task exists.

Gaffan's distinction between recognition memory and associative memory is less easy to apply to these tasks. Recognition memory would seem to be helpful to the animal during those tasks in which the animal either (a) experiences only the correct or incorrect cues or places during the registration period or (b) systematically experiences them in some temporal order so that they can be distinguished from the alternatives on the basis of recency. These conditions are usually fulfilled in direct DRT and DMTS tasks, but it is hard to see how they would apply to indirect DRT tasks such as those described in this paper. Even if recognition memory does play some role, however, it is hard to see why it should be operative in the distributed-cue condition and not during the clustered-cue condition.

\section{REFERENCES}

Alexinsky, T., \& Chapouthier, G. A new behavioral model for studying delayed response in rats. Behavioral Biology, 1978, 24, 442-456.

Coons, E. E., \& Miller, N. E. Conflict versus consolidation of memory traces to explain 'retrograde amnesia' produced by ECS. Journal of Comparative and Physiological Psychology, $1960,53,524-531$.

GafFAN, D. Loss of recognition memory in rats with lesions of the fornix. Neuropsychologia, 1972, 10, 327-341.

GafFAN, D. Recognition impaired and association intact in the memory of monkeys after transection of the fornix. Journal of Comparative and Physiological Psychology, 1974, 86, 1100-1109.

Horel, J. A. The neuroanatomy of amnesia. A critique of the hippocampal memory hypothesis. Brain, 1978, 101, 403-445.

Hunte R, W. S. The delayed reaction in animals and children. Behaviour Monographs, 1913, 2(Whole No. 6), 1-86.

JAMES, W. The principles of psychology. New York: Henry Holt, 1890.

McCord, F. The delayed reaction and memory in rats. I. Length of delay. Journal of Comparative Psychology, 1939, 27, 1-37.

MacCorquodale, K. An analysis of certain cues in the delayed response. Journal of Comparative and Physiological Psychology, $1947,40,239-253$.
MAIER, N. R. F. Delayed reaction and memory in rats. Journal of Genetic Psychology, 1929, 36, 538-549.

McGaugh, J. L., \& Herz, M. J. (EDs.). Memory consolidation. San Francisco: Albion, 1972.

O'KeEFE, J., \& Black, A. H. Single unit and lesion experiments on the sensory inputs to the hippocampal cognitive map. In J. Elliott \& J. Whelan (Eds.), Functions of the septo-hippocampal system. Amsterdam: Elsevier, 1978.

O'KeEfe, J., \& Conway, D. H. Hippocampal place units in the freely moving rat: Why they fire where they fire. Experimental Brain Research, 1978, 31, 573-590.

O'KeEFE, J., \& NADEL, L. The hippocampus as a cognitive map. Oxford: Clarendon Press, 1978.

O'Keefe, J., Nadel, L., Keightley, S., \& Kill, D. Fornix lesions selectively abolish place learning in the rat. Experimental Neurology, 1975, 48, 152-166.

Olton, D. S. Discrimination reversal performance after hippocampal lesions: An enduring failure of reinforcement and nonreinforcement to direct behavior. Physiology \& Behavior, 1972, 9, 353-356.

Olton, D. S., Becker, J. T., \& Handelman, G. Hippocampus, space and memory. Behavioral and Brain Sciences, 1979, 2, 313-365.

Olton, D. S., Becker, J. T., \& Handelman, G. E. Hippocampal function: Working memory or cognitive mapping? Physiological Psychology, 1980, 8, 239-246.

Olton, D. S., Walker, J. A., \& Gage, F. H. Hippocampal connections and spatial discrimination. Brain Research, 1978, 139, 295-308.

RESTLE, F. Discrimination of cues in mazes: A resolution of the "place-vs-response" question. Psychological Review, 1957, 64, 217-228.

Scoville, W. B., \& Milne R, B. Loss of recent memory after bilateral hippocampal lesion. Journal of Neurology, Neurosurgery and Psychiatry, 1957, 20, 11-21.

Sinnamon, H. M., Freniere, S., \& Kootz, J. Rat hippocampus and memory for places of changing significance. Journal of Comparative and Physiological Psychology, 1978, 92, 142-155.

\section{NOTES}

1. Strictly speaking, James was referring here to what he called secondary memory or memory proper. This was to be distinguished from primary memory, which lasted a short time after the event and was continuous with the event.

2. Of course we do-the lesion was not complete, etc., etc. 